\title{
Dynamic mathematical modelling of reaction kinetics for xylitol fermentation using Candida tropicalis
}

\begin{abstract}
Xylitol is an extracellular sugar alcohol produced by the biological conversion of xylose through the fermentation process. The present study investigates the reaction kinetics of xylitol fermentation by considering the effect of substrate and product concentration on the microbial growth rate. A $3.5-\mathrm{L}$ batch fermentation produced xylitol at different xylose concentrations and agitation speeds. The experimental data showed that the xylose concentration limit was less than $100 \mathrm{~g} / \mathrm{L}$ and that increasing the xylose concentration reduced the xylitol and cell yields. The optimum agitation speed was $400 \mathrm{rpm}$ with a kLa value of $32.6 \mathrm{hi} 1$. The experimental data were used to estimate the unknown parameters with gPROMS software. The Monod model was modified to include the inhibitory effect of the substrate and the limitation effect of dissolved oxygen on cell growth.
\end{abstract}

Keyword: Dynamic modelling; Fermentation; Growth kinetics; Yeast; Bioreactions 